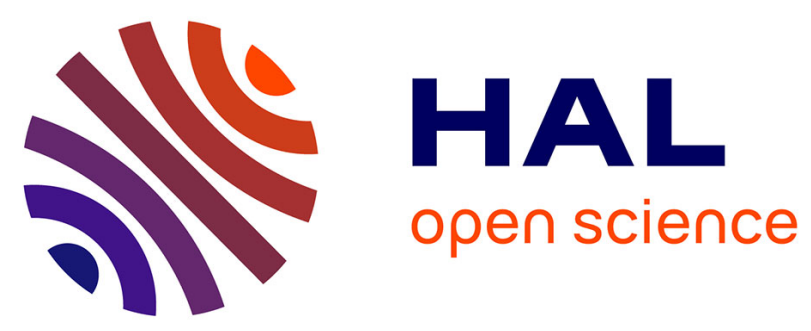

\title{
Optimal Power Allocation for Maximizing Network Lifetime in Wireless Sensor Networks
}

Said El Abdella Oui, Youssef Fakhri, Mérouane Debbah, Driss Aboutajdine

\section{To cite this version:}

Said El Abdella Oui, Youssef Fakhri, Mérouane Debbah, Driss Aboutajdine. Optimal Power Allocation for Maximizing Network Lifetime in Wireless Sensor Networks. ICMS 2012, May 2012, Tangier, Morocco. pp.511 - 516, 10.1109/ICMCS.2012.6320225 . hal-00771229

\section{HAL Id: hal-00771229}

https://hal-centralesupelec.archives-ouvertes.fr/hal-00771229

Submitted on 8 Jan 2013

HAL is a multi-disciplinary open access archive for the deposit and dissemination of scientific research documents, whether they are published or not. The documents may come from teaching and research institutions in France or abroad, or from public or private research centers.
L'archive ouverte pluridisciplinaire HAL, est destinée au dépôt et à la diffusion de documents scientifiques de niveau recherche, publiés ou non, émanant des établissements d'enseignement et de recherche français ou étrangers, des laboratoires publics ou privés. 


\section{Optimal Power Allocation for Maximizing Network Lifetime in Wireless Sensor Networks}

Said EL ABDELLA OUI*

LRIT, Unité Associée au CNRST (URAC 29)

Faculty of Sciences University

Mohammed V-Agdal

Rabat, Morocco.

elabdellaoui.said@yahoo.fr

\author{
Youssef FAKHRI \\ Équipe : Réseau x et Télécom., \\ Faculty of Sciences, Univ. Ibn Tofail, \\ Kenitra, Morocco. \\ LRIT, Unité Associée au CNRST \\ (URAC 29), FSR Univ. Moh. V \\ fakhri-y oussef@univ-ibntofail.ac.ma
}

\author{
Mérouane DEBBAH \\ Alcatel-Lucent Chair on \\ Flexible Radio, \\ Supelec, \\ Gif-sur-Yvette Cedex, \\ France \\ merouane.debbah@supelec.fr
}

\author{
Driss ABOUTAJDINE \\ LRIT, Unité Associée au CNRST \\ (URAC 29) \\ Faculty of Sciences University \\ Mohammed V-Agdal \\ Rabat, Morocco. \\ aboutaj@fsr.ac.ma
}

\begin{abstract}
In wireless sensor networks (WS N), Network lifetime is a critical metric, therefore, maximizing the network lifetime is a key challenge. In this article, we study the problem of optimal power allocation, taking into account the estimation of total signal-to-noise ratio (S NR) at the Fusion Center (FC). In order to analyze the proposed approach, we start by applying it in to orthogonal channels, and then we extend it to non-orthogonal channels. We consider that nodes transmit their data over quasistatic Rayleigh fading channels (QS RC) where these nodes have channel state Information (CSI). Simulations that have been conducted using these different channel conditions show the efficiency of the proposed approach.
\end{abstract}

Keywords; Orthogonal Channels; Non-Orthogonal Channels; Cooperative Communication; Convex Optimimisation; KKT.

\section{INTRODUCTION}

Wireless Sensor Networks (WSNs) have become recently the active research topics. The WSN is composed of several nodes distributed randomly in an area of interest where a node-source can transmit their data to the node-destination with the help of one or more relay nodes; cooperative communication [1]. These nodes supervise cooperatively the physical or environmental conditions, such as temperature, pressure, motion or pollutants at various locations [2]. In the conventional system model, the node works with an identical power resources, although this approach being clearly sub-optimal, therefore allocation power is necessary for a good energy management. In the literature, there are several works in this regard. In the next section; we give a brief overview on some previous works.

This paper is organized as follows. In the Section II, we give a comprehensive review of related work on network lifetime. Section III explains our method applied to the orthogonal channels and the non-orthogonal one considering that the nodes have direct access to the FC and the nodes transmit their data over a QSRC where the Channel State Information (CSI) is known by all the nodes. Section IV, presents the conducted experiments and the last section concludes the paper.

\section{RELATED WORK}

Several studies have addressed the problem of maximizing the network lifetime using various methods for minimizing energy consumption.
In this section, we present more detailed review of related works that try to prolong the network lifetime.

We begin by mentioning the work in [3], where Belmega et al. conclude that MIMO systems are more energy efficient than SISO systems if only the transmit power consumption is taken into account. However, when the circuitry energy consumption is considered, this conclusion is no longer true.

The limitation in a WSN is their physical size where they cannot carry multiple antennas at the same node. A new transmission technique named "Cooperative MIMO" has been introduced in [4][15]. This technique is based on the cooperation principle where the MIMO technology is virtually introduced.

In [5], the authors propose an optimal configuration algorithm for a multi-branch single-hop network where the total transmits power is minimized subject to a required error rate at the destination. In which, the transmission power of the source and all the relays are taken to be fixed and equal. In [6], the authors have given an extension for the previous work where considering a strategy to enhance the performance of previous algorithm. They have given an expression for the optimal allocation of transmission power amongst the selected relay nodes, whereby the total network power for a given system SER is minimized.

In [7] optimal solutions are presented for maximizing a static network lifetime through a graph theoretic approach using static broadcast tree.

In [8], the objective of the authors is to devise solutions that maximize network lifetime under such a minimal coverage constraint. Alfieri et al. proposed two in order to maximize the network lifetime: the first one, based on column generation, must run in a centralized way, whereas the second one is based on a heuristic algorithm aiming at a distributed implementation.

In [9], Nguyen et al. propose two techniques of cooperative reception to enhance energy efficiency. The whole space- time combination at the destination node is carried out by the first technique, while the second one independently performs signal processing and space-time combination at each cooperative node.

In [14], Li et al. introduce the low-energy adaptive clustering hierarchy (LEACH) framework to improve the 
energy efficiency where the STBC cooperative transmission is applied.

In this paper, we propose a power allocation strategy by reducing unnecessary consumption in order to maximize the lifetime of the entire network and thus ensure a desired Quality of Service (QoS) level. This work introduces a novel method for maximizing the sensor networks lifetime under unequal and time-varying channel conditions, taking into account the estimation of fusion center's SNR total quality constraint. The aim of this method is to provide the optimal operating point (transmission power) of the network to maximize its lifetime avoiding a large computational comple xity.

In order to analyze the proposed approach, we start by applying it in to orthogonal channels, and then we extend it to non-orthogonal channels. We consider that nodes transmit their data over quasi-static Rayleigh fading channels (QSRC) where these nodes have channel state Information (CSI).

\section{POWER ALLOCATION BY USINGTHE LAGRANGIAN AS OPTIMIZATION METHOD}

In this section, we will present our new method in order to maximize the network lifetime under the SNR total constraint at the Fusion Center (FC). We consider that nodes transmit their data over quasi- static Rayleigh fading channels (QSRC) where these nodes have channel state Information (CSI).

The analysis of our new approach starts with the orthogonal channel, and is then extend to the Non-Orthogonal one, making use of WSN's diversity to improve the QoS.

That is why, we assume $M$ sensors randomly distributed in the area of interest. When a monitored event occurs, the sensed observation made by an $\mathrm{i}^{\text {th }}$ sensor can be written as [10] (see figure 1):

$$
x_{i}=\theta+n_{i}
$$

Where $\theta$ is the source signal and $n_{i}$ is the additive complex Gaussian noise with $n_{i} \sim C N\left(0, \sigma^{2}{ }_{i t}\right)$. The observation $\mathrm{x}_{\mathrm{i}}$ is multiplied by the amplification gain $w_{i}$, and then transmitted

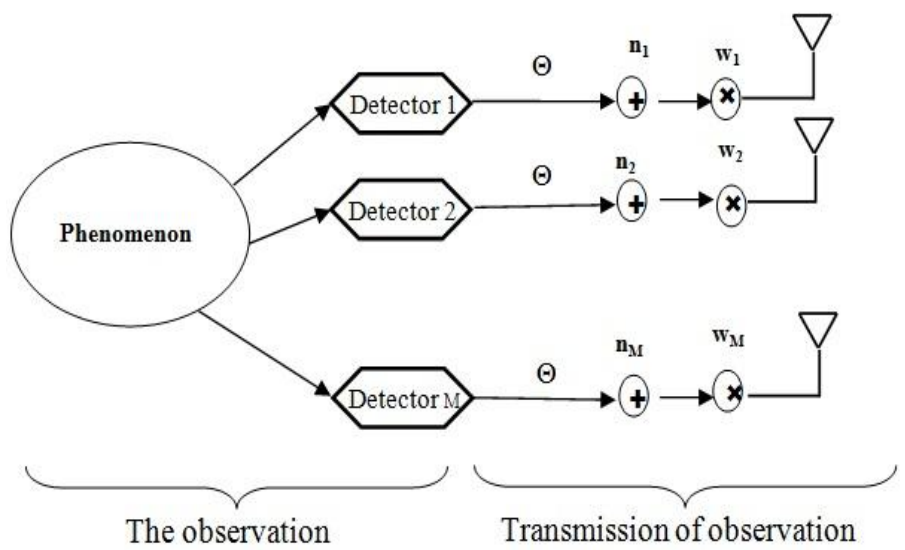

Figure 1: Observation system by the sensors to the FC. Therefore, the transmission power is written as $p_{i}=w_{i}^{2}\left(1+\sigma_{i t}^{2}\right)$ assuming that $E\left[\theta^{2}\right]=1$ where $E[:]$ is the mathematical expectation operator.

\section{A. Orthogonal Channels Configuration}

We consider the problem of optimal power allocation for WSNs when using orthogonal channels between each sensor and the FC taking into account that the nodes have Channel State Information (CSI). In addition, we suppose that a linear minimum mean square-error (LMMSE) detector is used at the receiver. This hypothesis will make easier the calculation of SNR at the FC, knowing that the latter is the sum of all the required SNRs fromeach sensor. In this transmission kind, we determine the SNR corresponding to each sensor in order to meet the overall SNR constraint ensuring that the sum of these SNRs is equal to the total SNR at the FC thanks to the use of the orthogonal channels.

\section{1) System Model}

We assume $\mathrm{M}$ sensors randomly distributed in the area of interest using orthogonal channels between the FC and each sensor (see figure 2).

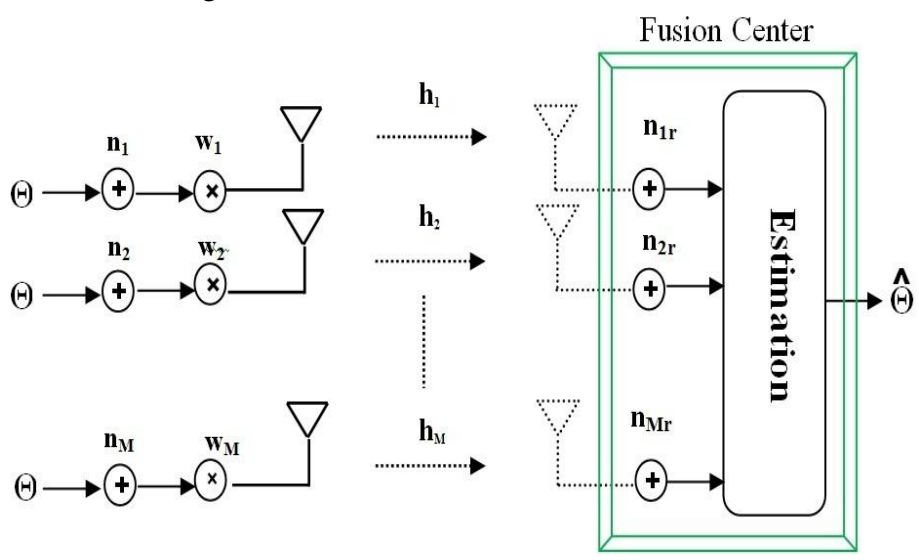

Figure 2: System model using orthogonal channels.

The received signal at the $\mathrm{FC}$ fro $\mathrm{m} \mathrm{i}^{\text {th }}$ sensor is defined by:

$$
y_{i}=h_{i} w_{i}\left(\theta+n_{i}\right)+n_{i r}
$$

Where $n_{i r}$ is the noise at the FC with a complex Gaussian distribution $n_{i r} \sim C \mathrm{~N}\left(0 ; \sigma_{i r}^{2}\right)$ and $h_{i}$ is the ith channel coefficient from the sensor $i$ to the FC. We assume that $\left|h_{i}\right|$ has a Rayleigh distribution.

$$
f\left(\left|h_{i}\right|\right)=\frac{\left|h_{i}\right| e^{\frac{-\left|h_{i}\right|^{2}}{2 \sigma_{h i}^{2}}}}{\sigma_{h i}^{2}}
$$

, where $\sigma_{h i}^{2}$ is known.

Assuming that we use real channels, the SNR corresponding to the $i^{\text {th }}$ sensor using the MMSE detector is given by (Figure 3):

$$
S N R_{i}=\frac{p_{s 2}}{p_{n 2}}=\frac{p_{s 1}\left|h_{i}\right|^{2}}{\sigma_{i r}^{2}+p_{n 1}\left|h_{i}\right|^{2}}=\frac{w_{i}^{2}\left|h_{i}\right|^{2} p_{\theta}}{\sigma_{i t}^{2} w_{i}^{2}\left|h_{i}\right|^{2}+\sigma_{i r}^{2}}
$$


Since $p_{\theta}=1$, then $\quad S N R_{i}=\frac{w_{i}^{2}\left|h_{i}\right|^{2}}{\sigma_{i t}^{2} w_{i}^{2}\left|h_{i}\right|^{2}+\sigma_{i r}^{2}}$

We express the $S N R_{i}$ in terms of transmission power, we obtain:

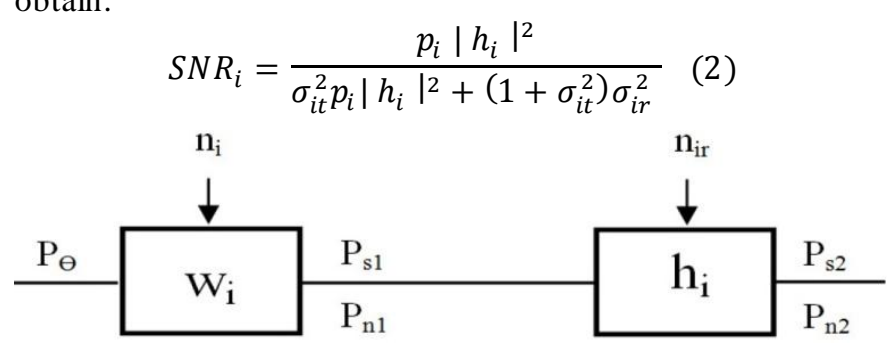

Figure 3: System model

Since we use the orthogonal channels, the total SNR at the FC will be the sum of all the required SNRs from each sensor. Then, the total SNR of all sensors at the FC can be written as follows:

$$
S N R=\sum_{i=1}^{M} S N R_{i}=\sum_{i=1}^{M}\left[\frac{p_{i}\left|h_{i}\right|^{2}}{\sigma_{i t}^{2} p_{i}\left|h_{i}\right|^{2}+\left(1+\sigma_{i t}^{2}\right) \sigma_{i r}^{2}}\right]
$$

Our aim is to maximize the lifetime of our network by taking into account the estimation of Fusion Center's SNR overall quality constraint.

\section{2) Problem Formulation:}

Our goal is to maximize the lifetime that is written as follows: $\quad \Lambda=\mathrm{N} * \mathrm{~T}$

Where the $\mathrm{T}$ is the period measurement of channel condition (we consider that $\mathrm{T}=1$ to simplify), and $\mathrm{N}$ is the number of transmissions before the network misses energy.

Hence, to maximize the lifetime of the network it is sufficient to maximize the number of transmissions for each sensor $\mathrm{N}$, taking into account the quality constraint defined as the estimation of the SNR at the FC being greater or equal than a target value $\gamma$. Then the formulation of our problem is:

$$
\left\{\begin{array}{c}
\operatorname{Max} N \\
E\left[\sum_{i=1}^{M} S N R_{i j}\right] \geq \gamma \quad j=1, \ldots \ldots, N \\
P_{i j} \geq 0 \quad \forall j
\end{array}\right.
$$

Since, it will be possible to decompose our problem into $M$ single convex optimization problems thanks to the use of the orthogonal channels. Thus, instead of maximizing the whole network lifetime, we will maximize the individual lifetime of each sensor ensuring that the sum of all the required SNRs of each sensor is equal to the total SNR at the FC. Using the weak law of large numbers [10] and maintaining that $\sum_{j}^{N} p_{i j}<\varepsilon_{i}$, we obtain:

$$
\left\{\begin{array}{c}
\operatorname{Max} N \\
\sum_{j=1}^{N} \sum_{i=1}^{M} S N R_{i j} \geq N \gamma \quad j=1, \ldots \ldots, N \\
P_{i j} \geq 0 \forall j
\end{array}\right.
$$

, where $\varepsilon_{i}$ is an initial energy for the $\mathrm{i}^{\text {th }}$ sensor. The estimated value of the SNR is the average SNR corresponding to the ith sensor at the FC over network lifetime, given by:

$$
\gamma_{i} \triangleq E\left[\frac{p_{i}\left|h_{i}\right|^{2}}{\sigma_{i t}^{2} p_{i}\left|h_{i}\right|^{2}+\left(1+\sigma_{i t}^{2}\right) \sigma_{i r}^{2}}\right]=E\left[S_{i}\right]
$$

It will be possible to decompose our problem into $\mathrm{M}$ single convex optimization problems thanks to the use of the orthogonal channels where the overall SNR at the FC is the sum of all the required SNRs for each sensor. Thus, instead of maximizing the whole network lifetime, we will maximize the individual lifetime of each sensor ensuring that the sum of all the required SNRs of each sensor is equal to the SNR at the FC. Hence, to maximize the individual lifetime it is adequate to minimize the transmission power. Therefore, the formulation of our problem becomes:

$$
\left\{\begin{array}{c}
\operatorname{Min} \sum_{j=1}^{N} P_{i j} \\
\sum_{j=1}^{N} S N R_{i j} \geq \quad N \gamma_{i} \quad j=1,, N \\
P_{i j} \geq 0 \forall j
\end{array}\right.
$$

Using the Lagrangian method, we obtain:

$$
\mathcal{L}(p, \lambda, v)=\sum_{j=1}^{N} p_{i j}+\sum_{j=1}^{N} \lambda_{i} p_{i j}
$$

$$
+v_{i}\left[N \gamma_{i}-\sum_{j=1}^{N} \frac{\left.\left.p_{i j}\right|_{i j}\right|^{2}}{\left.\left.\sigma_{i t}^{2} p_{i j}\right|_{i j}\right|^{2}+\left(1+\sigma_{i t}^{2}\right) \sigma_{i r}^{2}}\right] \text { (5) }
$$

The Karush-Kuhn-Tucker (KKT) Conditions [11] are as follows:

$$
\begin{gathered}
\lambda_{j} \geq 0, v_{i} \geq 0, \quad \lambda_{i} p_{i j}=0 \forall j \\
v_{i}\left[N \gamma_{i}-\sum_{j=1}^{N} \frac{p_{i j}\left|h_{i j}\right|^{2}}{\sigma_{i t}^{2} p_{i j}\left|h_{i j}\right|^{2}+\left(1+\sigma_{i t}^{2}\right) \sigma_{i r}^{2}}\right]=0 \\
\frac{\partial \mathcal{L}}{\partial p_{i j}}=1-\lambda_{j}+v_{i}\left[\frac{\sigma_{i r}^{2}\left|h_{i j}\right|^{2}\left(1+\sigma_{i t}^{2}\right)}{\left[\sigma_{i t}^{2} p_{i j}^{2}\left|h_{i j}\right|^{2}+\left(1+\sigma_{i t}^{2}\right) \sigma_{i r}^{2}\right]^{2}}\right]=0
\end{gathered}
$$

Taking into account this KKT conditions, we conclude that $v_{\mathrm{i}}>0$ and $\lambda_{\mathrm{j}}=0$. Then we obtain the following solution:

$$
\mathcal{P}_{i j}=\frac{\sqrt{\left(1+\sigma_{i t}^{2}\right) \sigma_{i d}^{2}}}{\sigma_{i t}^{2}\left|h_{i j}\right|^{2}}\left[\sqrt{\mathcal{V}_{i}}\left|h_{i j}\right|-\sqrt{\left(1+\sigma_{i t}^{2}\right) \sigma_{i r}^{2}}\right]
$$

Now, the challenge is to find the value of $v_{i}$, for this reason, we must express the $\gamma_{\mathrm{i}}=\mathrm{E}\left[\mathrm{S}_{\mathrm{i}}\right]$ in terms of the optimal power transmission scheme and then, derive the $E\left[p_{i}\right]$. The 
received SNR at the FC from the ith sensor during the jth transmission is given by:

$$
S_{i j}= \begin{cases}\frac{1}{\sigma_{i t}^{2}}-\frac{\alpha_{i}}{\sigma_{i t}^{2}} \frac{1}{\left|h_{i j}\right|} & \left|h_{i j}\right|>\alpha_{i} \\ 0 & \left|h_{i j}\right|<\alpha_{i}\end{cases}
$$

Where $\quad \alpha_{i}=\sqrt{\frac{\left(1+\sigma_{i t}^{2}\right) \sigma_{i r}^{2}}{v_{i}}}$

For calculate the $\mathrm{E}\left[\mathrm{S}_{\mathrm{i}}\right]$, we consider the following lemma [10] knowing that the random variable $\mathrm{Y}$ is defined in terms of another random variable $\mathrm{X}$ :

$$
\varphi= \begin{cases}C+g(x) & X>b \\ 0 & X<b\end{cases}
$$

Then, $\quad \varphi=C \int_{b}^{\infty} f(x) d x+\int_{b}^{\infty} f(x) g(x) d x$

, where $f(x)$ is the pdf of $X$.

Then, $\mathrm{E}\left[\mathrm{S}_{\mathrm{i}}\right]$ can be expressed by:

Then,

$$
E\left[S_{i}\right]=\frac{1}{\sigma_{i t}^{2}} \exp \left(\frac{-\alpha_{i}^{2}}{2 \sigma_{h i}^{2}}\right)-\frac{\alpha_{i}}{\sigma_{i t}^{2}} \int_{\alpha_{i}}^{\infty} \frac{1}{\sigma_{h i}^{2}} \exp \left(\frac{-x^{2}}{2 \sigma_{h i}^{2}}\right) d x
$$

$E\left[S_{i}\right]=\frac{1}{\sigma_{i t}^{2}} \exp \left(\frac{-\alpha_{i}^{2}}{2 \sigma_{h i}^{2}}\right)-\frac{\alpha_{i}}{\sigma_{i t}^{2}}\left[\frac{\sqrt{2 \pi}}{2 \sigma_{h i}}\left[1-\operatorname{erf}\left(\frac{\alpha_{i}}{\sqrt{2} \sigma_{h i}}\right)\right]\right]$

With erf $(\cdot)$ is an unilateral error function that is defined as follows:

Hence,

$$
\operatorname{erf}(x)=\frac{2}{\sqrt{\pi}} \int_{0}^{x} e^{-u^{2}} d u
$$

$$
E\left[P_{i}\right]=\frac{\sqrt{\pi} v_{i} \alpha_{i}}{\sqrt{2} \sigma_{h i} \sigma_{i t}^{2}}\left[1-\operatorname{erf}\left(\frac{\alpha_{i}}{\sqrt{2} \sigma_{h i}}\right)\right]-\frac{v_{i} \alpha_{i}^{2}}{2 \sigma_{i t}^{2} \sigma_{h i}^{2}} E_{1}\left[\frac{\alpha_{i}^{2}}{2 \sigma_{h i}^{2}}\right]
$$

, where $E_{n}=\int_{1}^{\infty} \frac{\exp (-x t)}{t^{n}} d t(\mathrm{x}>0, \mathrm{n}=0,1,2,3 \ldots \ldots)$ is the exponential integral function.

On the other hand, $\varepsilon_{\mathrm{i}}$ assuming that $\mathrm{T}=1$ s then $N=\frac{\varepsilon_{i}}{E\left[p_{i}\right]}(8)$

Then, the expected sensor lifetime can be determined as follows:

$$
N=\frac{\varepsilon_{i}}{\frac{\sqrt{\pi} \mathcal{V}_{i} \alpha_{i}}{\sqrt{2} \sigma_{h i} \sigma_{i t}^{2}}\left[1-\operatorname{erf}\left(\frac{\alpha_{i}}{\sqrt{2} \sigma_{h i}}\right)\right]-\frac{\mathcal{V}_{i} \alpha_{i}^{2}}{2 \sigma_{i t}^{2} \sigma_{h i}^{2}} E_{1}\left[\frac{\alpha_{i}^{2}}{2 \sigma_{h i}^{2}}\right]}
$$

\section{B. Non-Orthogonal Channels Configuration}

In this section, we assume that we have $M$ nodes transmit their data to the FC over a QSRC using the Non-Orthogonal channel taking into account that the nodes have Channel State Information (CSI) (see figure 4).

\section{1) System Model}

The received signal at the $\mathrm{FC}$ fro $\mathrm{m} \mathrm{i}^{\text {th }}$ sensor is defined by:

$$
y=\sum_{i=1}^{M} h_{i} w_{i}\left(\theta+n_{i}\right)+n_{r}
$$

Where $h_{i}$ is the channel coefficient from the sensor I to the FC such as these coefficients are i.i.d. We assume that $\left|h_{i}\right|$ has a Rayleigh distribution where $\sigma_{h i}^{2}$ represents the well known variance. Where,

$$
f\left(\left|h_{i}\right|\right)=\frac{\left|h_{i}\right| e^{\frac{-\left|h_{i}\right|^{2}}{2 \sigma_{h i}^{2}}}}{\sigma_{h i}^{2}}
$$

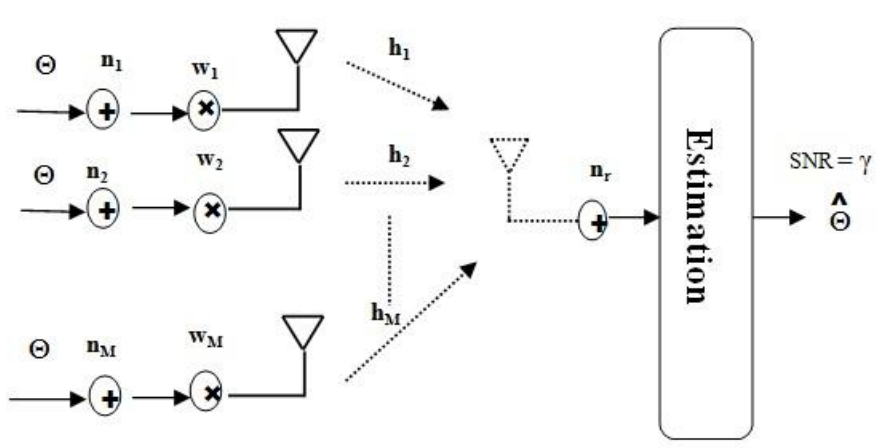

Figure 4: System model

Assuming that we use real channels and following the same lines as in last section, the SNR at the FC corresponding to $\mathrm{M}$ sensors using the MMSE detector is given by:

$$
S N R=\frac{\left(\sum_{i=1}^{M}\left|h_{i}\right| w_{i}\right)^{2}}{\sum_{i=1}^{M} w_{i}^{2}\left|h_{i}\right|^{2} \sigma_{i t}^{2}+\sigma_{r}^{2}}
$$

Our aim is to maximize the batteries lifetime duration while keeping the expected value of SNR greater than or equal to a target value $\gamma$.

\section{2) Problem Formulation}

At the lth instant, maximizing the lifetime relies on minimizing the power consumption, therefore the problem formulation is given as follows:

$$
\left\{\begin{array}{c}
\operatorname{Min} \sum_{i=1}^{M} w_{i}^{(l)^{2}}\left(1+\sigma_{i t}^{2}\right) \\
S N R^{(l)} \geq \gamma \quad l=1, \ldots, N \\
p_{i j} \geq 0
\end{array}\right.
$$

To find the optimal points, we use the Lagrange method while satisfying the constraints quoted before. The Lagrangian $£$ can be written as follows: 


$$
\begin{aligned}
\mathcal{L}\left(w_{i}^{(l)}, \lambda, v\right)= & \sum_{i=1}^{M} w_{i}^{\left({ }^{2}\right)^{2}}\left(1+\sigma_{i t}^{2}\right) \sum_{i=1}^{M} \lambda_{i} w_{i}{ }^{(l)} \\
& +v_{l}\left[\gamma\left[\sum_{i=1}^{M} w_{i}^{(l)}\left|h_{i}^{(l)}\right|^{2} \sigma_{i t}^{2}+\sigma_{r}^{2}\right]\right. \\
& \left.-\left[\sum_{i=1}^{M} w_{i}^{(l)}\left|h_{i}^{(l)}\right|\right]^{2}\right]
\end{aligned}
$$

Let consider the Karush-Kuhn-Tucker (KKT) Conditions for the problem:

$$
\left\{\begin{array}{c}
\lambda_{i} \geq 0, v_{l} \geq 0, \quad \lambda_{i} w_{i}^{(l)}=0 \forall l, i=1,2 \\
v_{l}\left[\gamma\left[\sum_{i=1}^{M} w_{i}^{(l)}{ }^{2}\left|h_{i}^{(l)}\right|^{2} \sigma_{i t}^{2}+\sigma_{r}^{2}\right]-\left[\sum_{i=1}^{M} w_{i}^{(l)}\left|h_{i}^{(l)}\right|\right]^{2}\right]=0 \\
\frac{\partial \mathcal{L}}{\partial w_{k}^{(l)}}=0
\end{array}\right.
$$

Then, the partial derivative of $£$ with respect to $\mathrm{w}_{\mathrm{k}}$ is:

$$
\begin{gathered}
\frac{\partial \mathcal{L}}{\partial w_{k}^{(l)}}=2 w_{k}^{(l)}\left(1+\sigma_{k t}^{2}\right)-\lambda_{k}+2 v_{l} \gamma\left|h_{k}^{(l)}\right|^{2} \sigma_{k t}^{2} w_{k}^{(l)} \\
-2 v_{l}\left|h_{k}^{(l)}\right|\left[\sum_{i=1}^{M} w_{i}^{(l)}\left|h_{i}^{(l)}\right|\right]=0
\end{gathered}
$$

Taking into account the KKT conditions, we find that $v_{l}>0$ and $\lambda_{k}=0$. Thus,

$$
w_{k}^{(l)}=\frac{v_{l}\left|h_{k}^{(l)}\right|\left(\sum_{i=1}^{M} w_{i}^{(l)}\left|h_{i}^{(l)}\right|\right)}{\left(1+\sigma_{k t}^{2}\right)+v_{l} \gamma\left|h_{k}^{(l)}\right|^{2} \sigma_{k t}^{2}}
$$

Where,

$$
\sum_{i=1}^{M} w_{i}^{(l)}\left|h_{i}^{(l)}\right|=\sum_{i=1}^{k-1} w_{i}^{(l)}\left|h_{i}^{(l)}\right|+\sum_{i=k+1}^{M} w_{i}^{(l)}\left|h_{i}^{(l)}\right|+w_{k}^{(l)}\left|h_{k}^{(l)}\right|
$$

To find the value of $\sum_{i=1}^{M} w_{i}^{(l)}\left|h_{i}^{(l)}\right|$ we replace (14) in (13), and it becomes:

$$
\begin{aligned}
& {\left[\sum_{i=1}^{M} w_{i}^{(l)}\left|h_{i}^{(l)}\right|\right]^{2}} \\
& =\frac{\gamma \sigma_{r}^{2}}{1-\gamma v_{l}^{2}\left[\sum_{i=1}^{M} \frac{\left|h_{i}^{(l)}\right|^{4}}{\left[\left(1+\sigma_{i t}^{2}\right)+v_{l} \gamma\left|h_{i}^{(l)}\right|^{2} \sigma_{i t}^{2}\right]^{2}} \sigma_{i t}^{2}\right]}
\end{aligned}
$$

Finally, equation (14) becomes:

$$
\frac{w_{k}^{(l)}=}{\left[\left(1+\sigma_{k t}^{2}\right)+v_{l} \gamma\left|h_{k}^{(l)}\right|^{2} \sigma_{k t}^{2}\right]\left[\sqrt{1-\gamma v_{l}^{2}\left[\sum _ { i = 1 } \left[h_{k}^{(l)} \mid \sigma_{r} \sqrt{\gamma} \frac{\left|h_{i}^{(l)}\right|^{4}}{\left.\left[1+\sigma_{i t}^{2}\right)+v_{l} \gamma\left|h_{i}^{(l)}\right|^{2} \sigma_{i t}^{2}\right]^{2}} \sigma_{i t}^{2}\right.\right.}\right]}
$$

Now, the challenge is to find the value of $v_{l}$.Therefore, we mu ltip ly equation (14) by $\left|\mathrm{h}_{\mathrm{k}}\right|$, we obtain:

$$
\left|h_{k}^{(l)}\right| w_{k}^{(l)}=\frac{v_{l}\left|h_{k}^{(l)}\right|^{2}\left(\sum_{i=1}^{M} w_{i}^{(l)}\left|h_{i}^{(l)}\right|\right)}{\left(1+\sigma_{k t}^{2}\right)+v_{l} \gamma\left|h_{k}^{(l)}\right|^{2} \sigma_{k t}^{2}}
$$

After that, we compute the sum of all the resulting equations, we obtain:

$$
\sum_{i=1}^{M}\left|h_{k}^{(l)}\right| w_{k}^{(l)}=\sum_{i=1}^{M} \frac{v_{l}\left|h_{k}^{(l)}\right|^{2}\left[\sum_{i=1}^{M} w_{i}^{(l)} h_{i}^{(l)}\right]}{\left(1+\sigma_{k t}^{2}\right)+v_{l} \gamma\left|h_{k}^{(l)}\right|^{2} \sigma_{k t}^{2}}
$$

Then,

$$
\sum_{i=1}^{M}\left|h_{k}^{(l)}\right| w_{k}^{(l)}\left[1-\sum_{i=1}^{M} \frac{v_{l}\left|h_{k}^{(l)}\right|^{2}}{\left(1+\sigma_{k t}^{2}\right)+v_{l} \gamma\left|h_{k}^{(l)}\right|^{2} \sigma_{k t}^{2}}\right]=0
$$

Since $\sum_{i=1}^{M} h_{k} w_{k}^{(l)} \neq 0$, we obtain:

$$
\sum_{i=1}^{M} \frac{\left|h_{k}^{(l)}\right|^{2}}{1+\sigma_{k t}^{2}\left(1+v_{l} \gamma\left|h_{k}^{(l)}\right|^{2}\right)}=\frac{1}{v_{l}}
$$

This equation is not written in a closed-form solution. So, it can be solved numerically using the function "Fminsearch" [12].

\section{EXPERIMENTAL RESULTS}

Several simulations have been conducted using MATLAB in order to compare and evaluate the behavior of both the Equal Power (EP) method [13] and our novel approach. For each simulation, we study the network lifetime while increasing the number of nodes. The simulations parameters are generated randomly such that each parameter $\mathrm{p}$ belongs to a uniform distribution between $\psi$ and $\varphi, p \in U[\psi ; \varphi]$. These parameters are summarized in the table of simulation parameters.

\section{A. Evaluation of the proposed method using orthogonal channels configuration}

Figure 5 shows the lifetime network while increasing the number of nodes using orthogonal channels. As it can be seen, the proposed approach improves EP method concerning the network lifetime. Actually, the network lifetime is extended by an average of $70,52 \%$ thanks to the use of the orthogonal 
channels where the overhead SNR at the FC is the sum of the SNRs coming from each sensor. Table 1 shows the parameters used for simulations.

TABLE I. SIMULATIONSPARAMETRES

\begin{tabular}{|c|l|}
\hline Estimate & Parameters \\
\hline $\mathrm{U}[0.1,0.4]$ & $\sigma_{h i}^{2}:$ The variances of channel estimation \\
\hline $\mathrm{U}[0.2,0.4]$ & $\sigma_{i r}^{2}:$ The noise variances at the FC \\
\hline $\mathrm{U}[0.02,0.2]$ & $\sigma_{i t}^{2}:$ The observation noise variances \\
\hline $\mathrm{U}[200,500]$ & $\varepsilon_{i}:$ The initial energy \\
\hline
\end{tabular}

\section{B. Evaluation of the proposed method using non-orthogonal channels configuration}

Figure 6 depicts the network lifetime while increasing the number of nodes. We can observe that using our new method the network lifetime is higher than that of EP method by an average of $82,80 \%$. Table 2 shows the parameters used for simu lations.

TABLE II. SIMULATIONS PARAMETRES

\begin{tabular}{|c|c|}
\hline Estimate & Parameters \\
\hline$U[0.1,0.4]$ & $\sigma_{h i}^{2}:$ The variances of channel estimation \\
\hline $\mathrm{U}[0.2,0.4]$ & $\sigma_{i r}^{2}:$ The noise va riances at the FC \\
\hline $\mathrm{U}[0.02,0.2]$ & $\sigma_{i t}^{2}:$ The observation noise va riances \\
\hline $\mathrm{U}[200,500]$ & $\varepsilon_{i}:$ The initial energy \\
\hline
\end{tabular}

\section{CONCLUSION}

This paper presents a new algorithm which aims to minimize the power consumption and consequently maximize the network lifetime. This method takes into consideration the estimation of the overall SNR at the FC.

Our simulations show that our new method using the orthogonal channels scheme and Non-Orthogonal one, consumes less energy than the EP method. The future work is to apply our new method to Non-Orthogonal channels assuming that the channel coefficients are unknown.

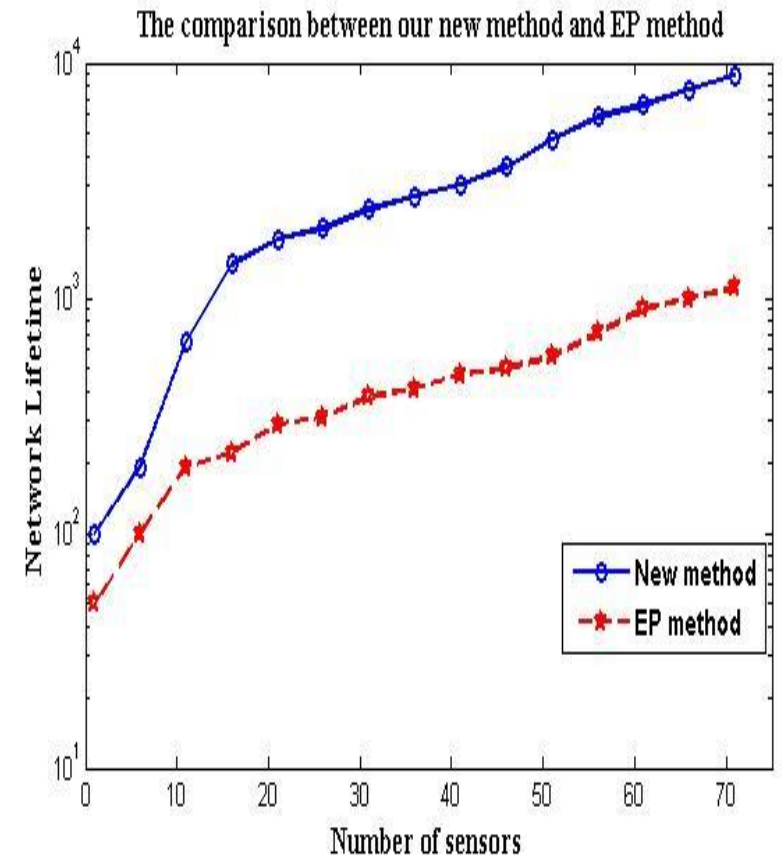

Figure 6: Network lifetime using the Non-orthogonal channels

\section{REFERENCES}

[1] A. Sendonaris, E. Erkip, and B. Aazhang, "User Cooperation Diversity Part I and Part II," IEEE Trans. Commun., vol. 51, no. 11, pp. 1927-48, Nov. 2003

[2] J. Liu, F. Zhao, D. Petrovic, "Information-Directed Routing in Ad Hoc Sensor Networks", IEEE Journal on Selected Areas in Communications, vol. 23, no. 4, pp. 851-861, April 2005.

[3] L. Collin, O. Berder, P. Rostaing, and G. Burel, "Optimal Minimum Distance Based Precoder for MIMO Spatial Multiplexing Systems," IEEE Transactions on Signal Processing, vol. 52, no. 3, pp. 617-627, Mar. 2004.

[4] E. V. Belmega, S. Lasaulce, and M. Debbah, "A survey on energyefficient communications," IEEE Intl. Symp.on Personal, Indoor and Mobile Radio Communications (PIMRC 2010), Istanbul, Turkey, invited paper, pp. 289, Sep. 2010.

[5] S. Cui, A. J. Goldsmith, and A. Bahai, "Energyefficiency of MIMO and cooperative MIMO techniques in sensor networks," IEEE Jour. On Selected Areas in Communications, vol. 22, no. 6, pp. 1089-1098, Aug. 2004.

[6] J. Winters: The diversity gain of transmit diversity in wireless systems with Rayleigh fading, IEEE Transactions on Vehicular Technology, Vol 47, No. 1, pp. 119-123, 1998

[7] J. Vazifehdan, H. Shafiee, "Multi-Branch Configuration of Dynamic Wireless Cooperat ive Networks, ICSPC, Dubai, UAE, 2007.

[8] Y. Thomas Hou, Y. Shi, D. Hanif Sherali and E. Jeffrey Wieselthier: Multicast Communications in Ad HocvNetworks Using Directional Antennas: A Lifetime-Centric Approach, IEEE Transactions on Vehicular Technology, Vol. 56, No. 3, 2007.

[9] T. Nguyen, O. Berder, and O. Sentieys, 'Impact of transmission synchronization error and cooperative reception techniques on the performance of cooperative MIMO systems," IEEE International Conference in Communications, Beijing, China, May 2008.

[10] F. Namin and A. Nosratinia, "Progmatic Lifetime maximization of cooperative Sensor Networks via a decomposition approoch," in Acoustics, Speech and Signal Processing- Las Vegas, Nevada, U.S.A, no .4 , pp. 3017, Apr. 2008

[11] H. Wei, H. Sasaki, and j. Kubokawa, "A decoupled solution of hydrothermal optimal power flow problem by means of interior point method and network programming," IEEE Transactions on Power Systems, Vol. 13, no. 2, pp. 286-293, May 1998.

[12] http://www.mathworks.com/help/techdoc/ref/fminsearch.html

[13] H. Goudarzi, M. R. Pakravan, 'Equal Power Allocation scheme for cooperative diversity," in 4th IEEE/IFIP International Conference, Tashkent- uzbekistan, pp. 1-5,Sept. 2008.

[14] X. Li, M. Che, and W. Liu, "Application of ST BC-ecoded cooperative transmissions in wireless sensor networks", IEEE Signal Processing Letters, vol. 12, no. 2, Feb. 2005.

[15] S. E. Abdellaoui, Y. Fakhri, and D. Aboutajdine, "Power and Throughput Optimization in Ad-hoc Cooperative MIMO Systems," International Conference in Multimedia Computing and Systems, Ouarzazzate Morocco, no. 8, pp. 273-277, Av. 2009.

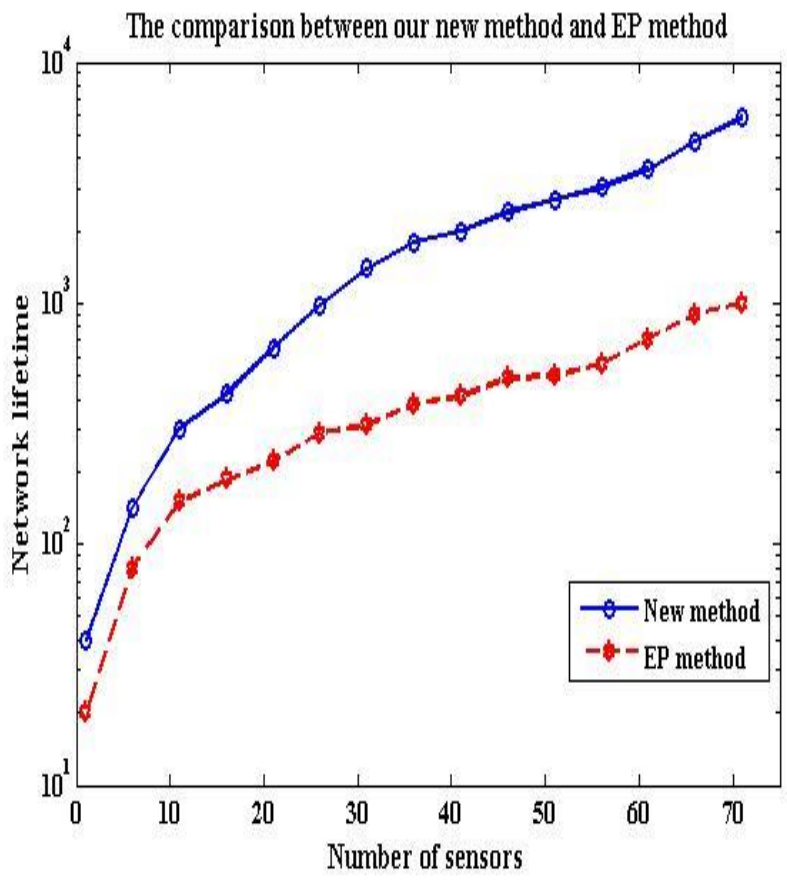

Figure 5: Network lifetime using the orthogonal channels 